\title{
Prolonged human neural stem cell maturation supports recovery in injured rodent CNS
}

\author{
Paul Lu, ${ }^{1,2}$ Steven Ceto, ${ }^{2,3}$ Yaozhi Wang, ${ }^{2}$ Lori Graham, ${ }^{2}$ Di Wu, ${ }^{2}$ Hiromi Kumamaru, ${ }^{2}$ Eileen Staufenberg, ${ }^{2}$ and Mark H. Tuszynski ${ }^{1,2}$ \\ 'VA San Diego Healthcare System, San Diego, California, USA. Department of Neurosciences and ${ }^{3}$ Biomedical Sciences Graduate Program, UCSD, La jolla, California, USA.
}

\begin{abstract}
Neural stem cells (NSCs) differentiate into both neurons and glia, and strategies using human NSCs have the potential to restore function following spinal cord injury (SCI). However, the time period of maturation for human NSCs in adult injured CNS is not well defined, posing fundamental questions about the design and implementation of NSC-based therapies. This work assessed human H9 NSCs that were implanted into sites of $\mathrm{SCl}$ in immunodeficient rats over a period of 1.5 years. Notably, grafts showed evidence of continued maturation over the entire assessment period. Markers of neuronal maturity were first expressed 3 months after grafting. However, neurogenesis, neuronal pruning, and neuronal enlargement continued over the next year, while total graft size remained stable over time. Axons emerged early from grafts in very high numbers, and half of these projections persisted by 1.5 years. Mature astrocyte markers first appeared after 6 months, while more mature oligodendrocyte markers were not present until 1 year after grafting. Astrocytes slowly migrated from grafts. Notably, functional recovery began more than 1 year after grafting. Thus, human NSCs retain an intrinsic human rate of maturation, despite implantation into the injured rodent spinal cord, yet they support delayed functional recovery, a finding of great importance in planning human clinical trials.
\end{abstract}

\section{Introduction}

Neural stem cells (NSCs) differentiate into neurons and glia and can potentially replace lost neural systems after central nervous system (CNS) injury (1-8). When implanted into sites of spinal cord injury (SCI), rat- and human-derived multipotent neural progenitor cells, human embryonic stem cell-derived (ESC-derived) NSCs, and human induced pluripotent stem cell-derived (iPSC-derived) NSCs all extend very large numbers of axons over long distances and form synapses with host neurons $(4,5)$ that in some cases support functional improvement $(4,8-10)$. These findings suggest the potential for human translation; indeed, NSC clinical trials for SCI have begun, and more are planned (ClinicalTrials.gov).

Yet a question of great relevance to the design, implementation, and performance of clinical trials remains: at what rate will human NSCs mature when grafted to sites of SCI? This is important for several reasons: if human NSCs mature rapidly, then it is reasonable to assess outcomes on a typical clinical trial time frame of months. In contrast, if NSC maturation parallels normal human development and cells mature over much longer time periods (see below), then clinical trials must be designed over very extended time periods. Prolonged maturation in turn raises fundamental biological questions: will injured host axons and neurons remain responsive to NSC grafts if the latter are mature and capable of

Related Commentary: p. 3284

Conflict of interest: The authors have declared that no conflict of interest exists.

Submitted: January 20, 2017; Accepted: June 27, 2017.

Reference information: / Clin Invest. 2017;127(9):3287-3299.

https://doi.org/10.1172/JCI92955. sustaining function only months or even years after grafting? If chronically injured host axons retract from injury sites over the time period that human grafts are maturing, can neural circuitry be reconstituted? If NSC-derived axons are pruned over time (as during neural development), might functional benefits be lost?

The human gestational period is 280 days; rat gestation is 21 days (11). At birth, the human brain roughly corresponds to a postnatal day 7 rat brain based on body/brain weight ratios, and a 2- to 3-year-old human brain corresponds to a postnatal day-20 rat brain (11). Yet most NSC grafting studies have been performed over relatively short survival times of weeks to a few months in vivo $(3,5,9,12)$. Moreover, it is possible that the rodent environment, especially the adult traumatic environment, will alter the fate or rate of human NSC maturation; these questions have, until the present, remained unanswered. Previous studies have examined the differentiation and maturation of human fetus-derived NSCs (13), oligodendrocyte progenitors $(14,15)$, glial progenitor cells $(6,16)$, or human ESC-derived neurons (17, 18); however, none of these studies examined human neural cell maturation in an adult rodent CNS trauma model. In addition, human cells were transplanted either into embryonic or neonatal rodents, but not into adult animals; the developing CNS environment is distinct from the adult CNS, potentially influencing the differentiation and maturation of human NSCs. An assessment of the fate and maturation of human ESC-derived NSCs in adult lesion models is important not only for stem cell biology, but also for the practical and realistic design of translational human trials involving NSCs for SCI and other human disorders. Accordingly, we examined the nature and time course of human NSC maturation in adult rats that underwent SCI. Post-grafting time periods ranged from 1 month to 1.5 years. 

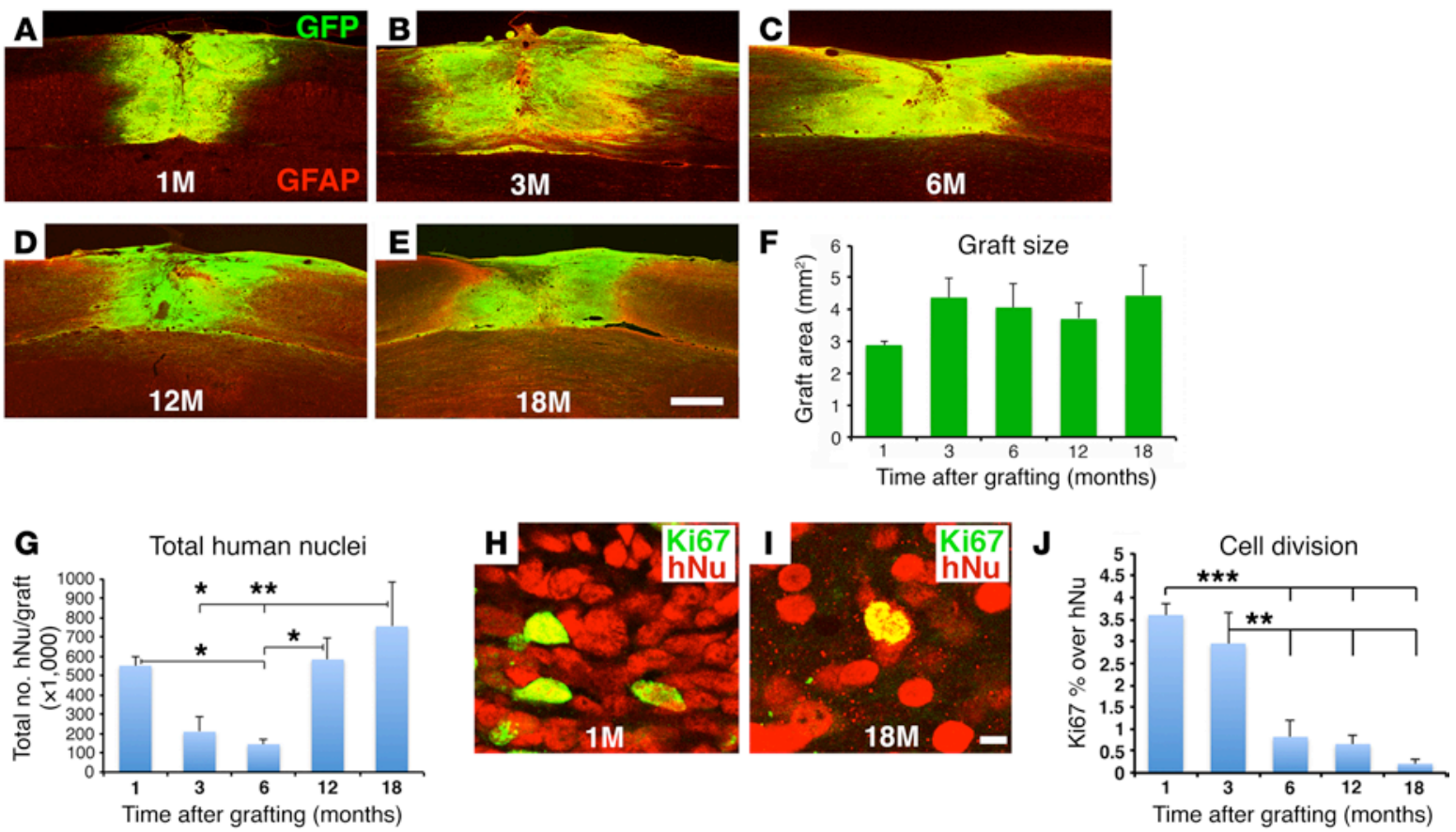

Figure 1. H9-NSC graft morphology and Ki67 immunolabeling. (A-E) Graft size was stable over time in the C5 hemisection lesion site, and grafts were well integrated with the host. GFP and GFAP double-labeling (horizontal sections). (F) Grafts nonsignificantly expanded from 1 to 3 months after grafting $(P=0.6$, by ANOVA) and were stable in size thereafter. Data represent the mean \pm SEM. (G) The total number of grafted human cells (detected by hNu, a human-specific cell marker) was significantly reduced at 3 and 6 months, but recovered by 12 and 18 months. $P<0.05$, by ANOVA and ${ }^{*} P<0.001$ and ${ }^{*} P<0.05$, by Fisher's exact post-hoc test. Data represent the mean \pm SEM. (H-J) Cell proliferation was significantly reduced after 3 months. hNu indicates the human-specific nucleus marker; Ki67 labels proliferating cells. $P<0.0001$, by ANOVA and ${ }^{* * *} P<0.001$ and ${ }^{* *} P<0.01$, by Fisher's exact posthoc test comparing results at 1 and 3 months with results at 6,12 , and 18 months, respectively. Data represent the mean \pm SEM. For F, G, and J: 1 month, $n=3 ; 3$ months, $n=3 ; 6$ months, $n=5 ; 12$ months, $n=3$; and 18 months, $n=4$. Scale bars: $550 \mu \mathrm{m}(\mathbf{A}-\mathbf{E}) ; 7 \mu \mathrm{m}(\mathbf{H}$ and $\mathbf{I})$.

\section{Results}

We used the widely available H9 human NSC line, derived from human ESCs (Gibco Human Neural Stem Cells) $(19,20)$. This NSC line is well characterized: it expresses the NSC-specific markers nestin and SOX2 and differentiates into neurons, oligodendrocytes, and astrocytes (21). H9 NSCs were transduced to express GFP and were harvested and embedded in fibrin matrices containing a growth factor cocktail (see Methods and ref. 4) to promote NSC survival and retention in the lesion site. Cells were transplanted into C5 spinal cord lateral hemisection lesion sites in immunodeficient rats (total $n=18$ ) 2 weeks after the SCI. Right hemisections are large lesions that entirely remove the right half of the spinal cord, thereby impairing function of the ipsilateral forelimb (22). Control rats $(n=5)$ underwent the same hemisection lesions and injections of the fibrin matrix containing the growth factor cocktail, without NSCs. NSC-grafted subjects were perfused at serial time points after grafting: 1 month $(n=3) ; 3$ months $(n=3) ; 6$ months $(n=5)$; 12 months $(n=3)$; and 18 months $(n=4)$. Control subjects were perfused 12 months after lesioning. Behavioral analysis of the affected forelimb was conducted monthly for those NSCgrafted subjects that survived for $12(n=7)$ or $18(n=4)$ months and control subjects $(n=5)$ that survived for 12 months.

H9 NSC graft size is stable over time. GFP-expressing human NSCs survived well and filled C5 hemisection sites at each survival time point (Figure 1, A-E). Graft size tended to increase from 1 to 3 months, but was stable thereafter (Figure $1 \mathrm{~F}$ ). The total number of grafted human cells, assessed by the human-specific nuclear marker hNu, was maximal 1 month after grafting, declined at 3 and 6 months after grafting, and then gradually recovered by 12 and 18 months (Figure $1 \mathrm{G})$. Double-labeling of grafted cells for $\mathrm{hNu}$ and the cell proliferation marker Ki67 demonstrated that 3.6\% $\pm 0.2 \%$ and $3.0 \% \pm 0.7 \%$ of grafted human cells were proliferating 1 and 3 months after grafting, respectively, but this number was significantly and substantially reduced to $0.8 \% \pm 0.4 \%$ and $0.7 \% \pm 0.2 \%$ by 6 and 12 months after grafting, respectively (Figure $1, \mathrm{H}-\mathrm{J})$. Cell division was further attenuated by 18 months after grafting to only $0.2 \% \pm 0.1 \%(P<0.0001$, by ANOVA; $P<0.01$, by Fisher's exact post-hoc test comparing 18 months with 1 and 3 months, respectively) (Figure 1, H-J). Indeed, we detected no Ki67 labeling in 2 of 4 subjects 18 months after grafting. Approximately $70 \%$ of dividing Ki67-labeled cells colocalized with human-specific nestin, indicating that many dividing cells in grafts were dividing NSCs (Supplemental Figure 1; supplemental material available online with this article; https://doi.org/10.1172/ JCI92955DS1). Some of these dividing NSCs were clustered, as was seen with NSC niches (23). These data indicate that grafted H9 human NSCs became nearly entirely postmitotic, without tumor formation, in lesion sites at long post-grafting time periods, an important safety consideration for human clinical application.

Grafted human neurons mature gradually over 18 months. One month after grafting, human NSCs densely expressed the immature neuronal marker doublecortin (DCX) (Figure 2A), but not the 

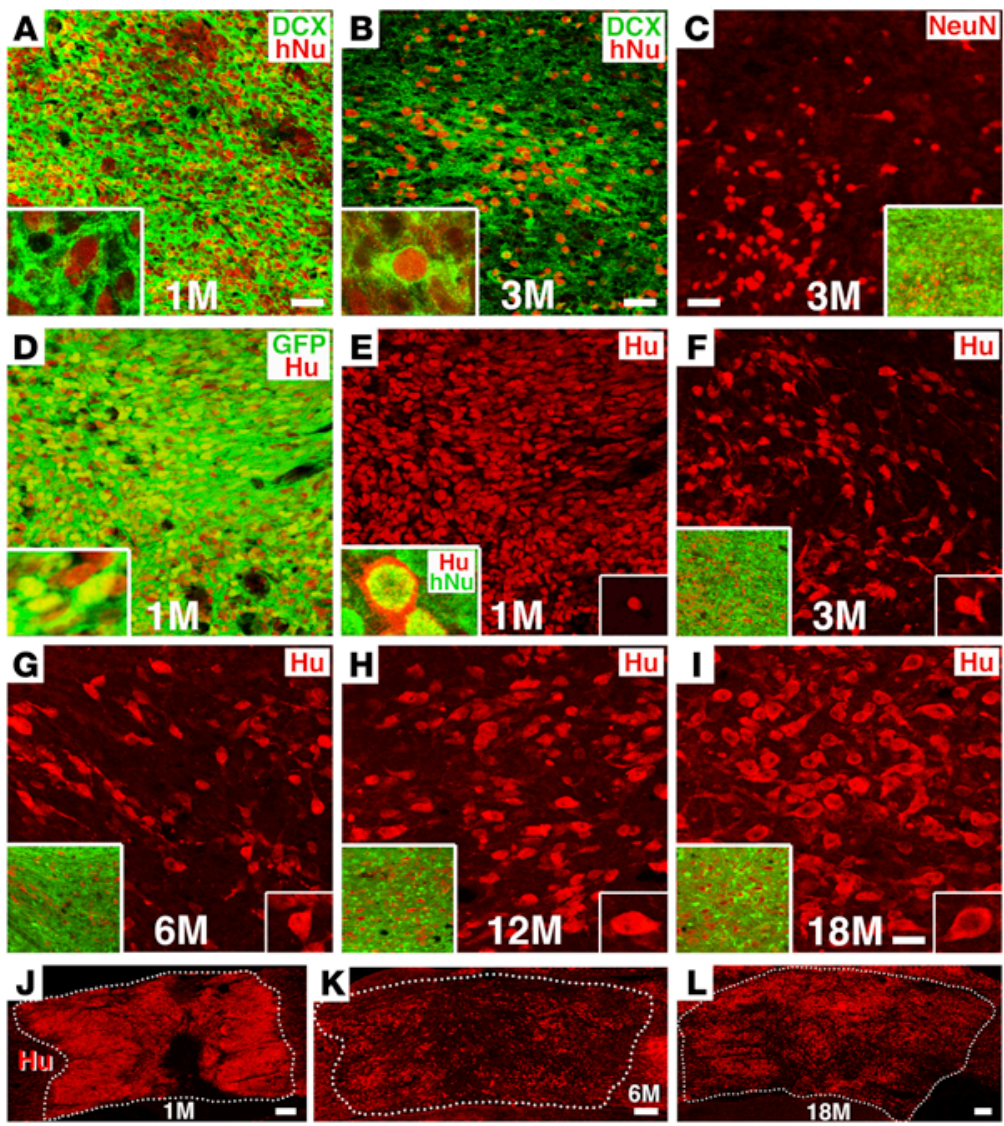

M

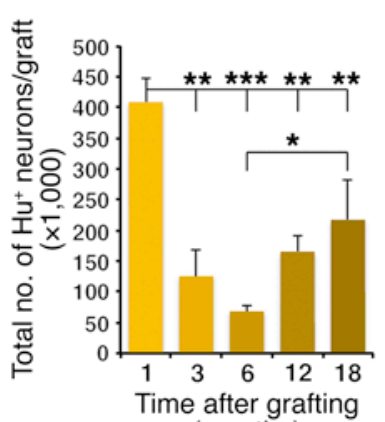

(months)
$\mathbf{N}$

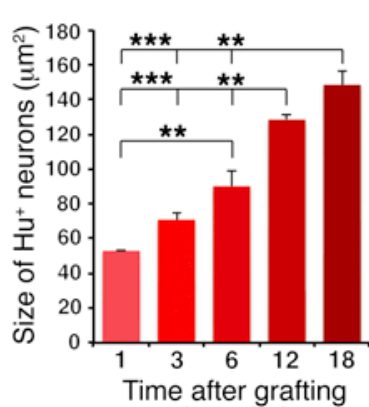

(months)
0

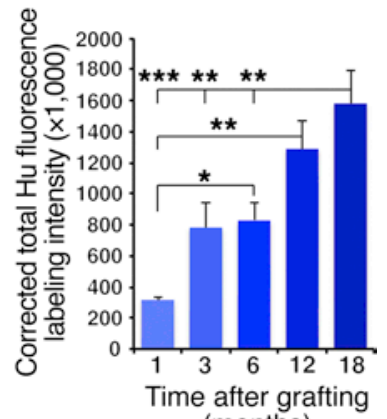

(months)
Figure 2. Neuronal maturation over time. (A) One month after grafting, human H9-NSCs expressed the human nuclear marker $\mathrm{hNu}$; many cells also expressed the immature neuronal marker DCX in the cytoplasm. Inset shows image at higher magnification and colocalization of hNu and DCX. (B) Expression of DCX was substantially reduced by 3 months after grafting, and (C) at this time point, cells first express NeuN. Inset images show higher magnification.

(D-I) Expression of the pan-neuronal marker Hu from 1 to 18 months after grafting. Insets show higher magnification. Many NSCs, labeled for GFP, also expressed Hu at 1 month. At 3 and 6 months, $\mathrm{Hu}^{+}$cell numbers were substantially reduced and partially recovered by 12 and 18 months (quantified in $\mathbf{M}$ ). (J-L) Hu cell distribution in the graft at 1, 6, and 18 months. Hu cell size increased over time (quantified in $\mathbf{N}$ ) and adopted a more mature neuronal morphology. (0) Quantification of Hu fluorescence labeling intensity over time. All data represent the mean \pm SEM. $P<0.001$, ANOVA $(\mathbf{M}-\mathbf{0})$ and ${ }^{*}{ }^{*} P<0.001,{ }^{* *} P<0.01$, and ${ }^{*} P<0.05$, by Fisher's exact post-hoc comparison test for $1(n=3), 3(n=3), 6(n=5), 12$ $(n=3)$, and 18 months $(n=4)$. Scale bars: $32 \mu \mathrm{m}(\mathbf{A}-\mathbf{I}) ; 250$ $\mu \mathrm{m}(\mathrm{J}-\mathrm{L})$. Original magnification of Images in insets: A, B, D, and E: $\times 1200 ; \mathbf{C}, \mathbf{F}-\mathbf{I}: \times 400$. mature neuronal marker NeuN. By 3 months, we observed that DCX expression in grafts was reduced, and at 6 months and thereafter was restricted to small subregions of grafts (Figure 2B and Supplemental Figure 2, A-C). NeuN expression was first evident 3 months after grafting and persisted thereafter (Figure 2C and Supplemental Figure 2, D-F). Notably, the number, size, and intensity of NeuN-expressing cells in grafts appeared to increase from 3 months to 1 year after grafting, an observation that was confirmed on quantification (Figure 2C and Supplemental Figure 2, D-F and J-L). Thus, neurons exhibited evidence of gradual maturation over a full year after grafting.

To assess changes in the total number of grafted neurons over time, we used the specific neuronal marker $\mathrm{Hu}$, which is expressed by all neurons, including both immature and mature postmitotic neurons (24-26) and is distinct from the hNu antibody, which specifically labels human nuclei but is not restricted to neurons (Figure 2, A, B, E inset, and Supplemental Figure 2, G-I). We found that Hu labeled all immature neurons that expressed DCX both in vitro and in vivo (Supplemental Figure 3, A-C, and G-I) and all NeuN-expressing neurons both in vitro and in vivo (Supplemental Figure 3, D-F and J-L). Using $\mathrm{Hu} / \mathrm{hNu}$ double labeling, we quantified the total number of grafted neurons, neuron size, and $\mathrm{Hu}-$ labeling intensity over time.

The proportion of all grafted cells that expressed the neuronal marker $\mathrm{Hu}(\mathrm{Hu} / \mathrm{hNu})$ steadily decreased over time to 1 year and then stabilized: $76 \% \pm 4 \%$ of all cells expressed $\mathrm{Hu}$ at 1 month; $60 \%$ $\pm 5 \%$ at 3 months; $49 \% \pm 3 \%$ at 6 months; $29 \% \pm 2 \%$ at 12 months; and $29 \% \pm 3 \%$ at 18 months, a gradual reduction that was significant $(P<0.0001$, by 1 -way ANOVA). Interestingly, the total number of grafted human NSCs expressing the neuronal marker $\mathrm{Hu}$ was maximal 1 month after grafting, as assessed using semistereological methods (Figure 2, D, E, J, and M). At later time points, as neurons matured, the number of neurons expressing Hu had a $70 \%$ 

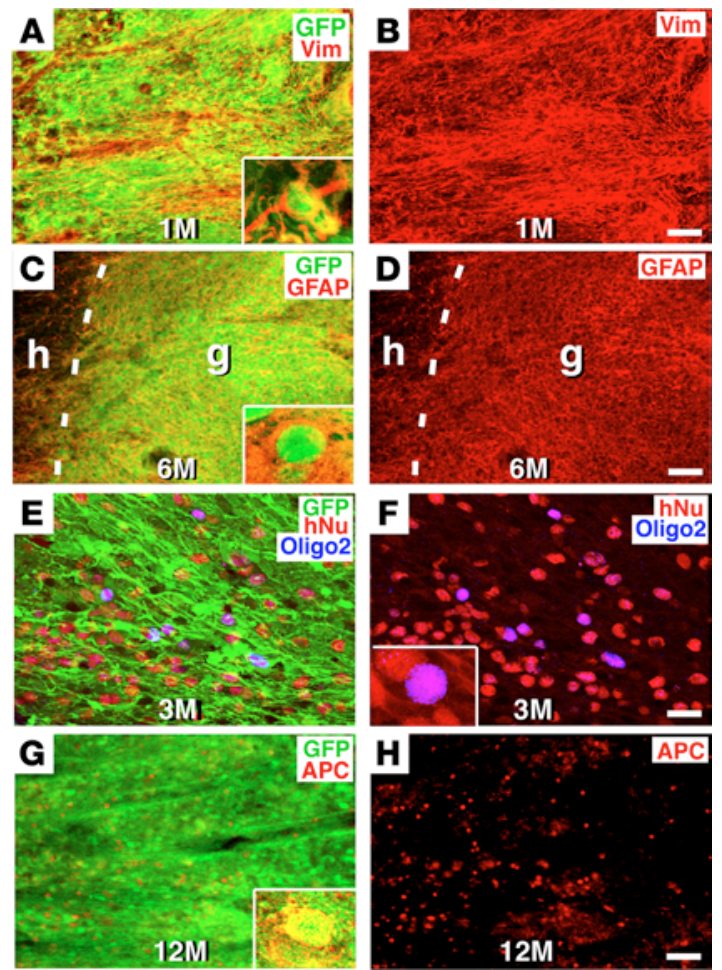

reduction 3 months after grafting $(P<0.001$, by 1 -way ANOVA; $P<$ 0.001 vs. 1 month after grafting, by Fisher's exact post-hoc test) and an $83 \%$ reduction 6 months after grafting $(P<0.0001$ vs. 1 month after grafting, by Fisher's exact post-hoc test) (Figure 2, F, G, K, and M). Yet after 6 months, we found that the number of grafted human neurons expressing $\mathrm{Hu}$ progressively recovered: at 12 months, cell numbers increased by 2.4-fold compared with the numbers at 6 months and further increased by 3.2 -fold at 18 months $(P<0.05$ vs. 6 months after grafting, by Fisher's exact post-hoc test) (Figure 2, $\mathrm{H}$, $\mathrm{I}, \mathrm{L}$, and $\mathrm{M}$ ). The final total number of grafted cells expressing $\mathrm{Hu}$ after 18 months represented $53 \%$ of the original number of Hu cells at 1 month (Figure 2, I, L, and M). Similarly, quantification of the number of neurons expressing the mature neuronal marker NeuN was lowest at 3 and 6 months and increased at 12 and 18 months (Figure 2C and Supplemental Figure 2, D-F, and J). As expected, the total number of NeuN-labeled cells was lower than the number of $\mathrm{Hu}$-labeled cells, because $\mathrm{Hu}$ detects both immature and mature neurons (25) (Figure 2, C-L, and Supplemental Figures 2 and 3), and because NeuN does not recognize all classes of mature neurons (27). These findings indicate that grafted NSCs undergo dynamic neurogenesis: neuron numbers are maximal 1 month after grafting and then exhibit a delayed phase of reduction 3-6 months after grafting, followed by gradual recovery. DAPI staining at 3 and 6 months confirmed the presence of nuclear fragmentation in some grafted human cells, consistent with apoptotic cell death (28) (Supplemental Figure 4). As noted above, nearly 1\% of grafted cells continued to express the cell division marker Ki67 through 1 year after grafting (Figure 1J), and small amounts of cells expressed DCX through 18 months (Figure 2, A and B, and Supplemental Figure 2, $\mathrm{A}-\mathrm{C}$ ), constituting a newly dividing cell pool that may reconstitute neuron numbers; only $0.2 \%$ of cells expressed Ki67 by 18 months, a point at which neuron numbers had stabilized.
Figure 3. Glial maturation over time. (A and B) GFP and vimentin (Vim) immunolabeling revealed colocalization of grafted human cells expressing GFP with the glial progenitor marker vimentin 1 month after grafting. Inset in $\mathbf{A}$ is a higher-magnification image. (C and $\mathbf{D})$ The mature astrocyte marker GFAP was not detectable in human NSC grafts until 6 months after implantation. Dashed lines indicate the host/graft (h/g) interface. Inset in $\mathbf{C}$ is a higher magnification image showing colocalization of GFP and GFAP. (E and F) The oligodendroglial marker Oligo2 was first detectable 3 months after grafting and colocalized with GFP and hNu markers. $(\mathbf{G}$ and $\mathbf{H})$ However, the more mature oligodendrocyte marker APC was first detected only 12 months after NSC grafting and colocalized with GFP (inset in G). Scale bars: $60 \mu \mathrm{m}$ (A-D, G, and $\mathbf{H}) ; 24 \mu \mathrm{m}$ (E and F). Original magnification of images in insets $(\mathbf{A}, \mathbf{C}, \mathbf{F}, \mathbf{C}): \times 600$.

Neuronal hypertrophy is an indication of continuing cell maturation (29). While total neuron numbers dropped and then recovered over time, mean Hu-labeled neuron size gradually and significantly increased by 3 -fold over successive post-grafting time points through 12 months $(P<0.0001$, by ANOVA) (Figure 2N). Moreover, the mean intensity of Hu neuronal reaction product per neuron also increased over time $(P<0.001$, by 1 -way ANOVA) (Figure 2O). Cells also gradually adopted a more mature, pyramid-like morphology over time (Figure 2, D-I). We observed parallel changes in size and intensity of NeuN-labeled cells (Figure 2C and Supplemental Figure 2, D-F, K, and L).

Collectively, the preceding data indicate that, following a 1- to 3-month period of delayed cell loss, grafted neurons will mature steadily over the ensuing 12 months. This time period corresponds to a normal human time course of neuronal maturation, despite graft placement into an injured adult rodent environment.

The spatial distribution of neurons in the graft also evolved over time. One month after grafting, cells were relatively homogeneously distributed in the lesion site (Figure 2, A, D, E, and J). However, by 3 and 6 months, when we observed extensive loss of cells expressing neuronal markers, cell distribution in the graft was uneven, with some regions containing clusters of $\mathrm{Hu}$-expressing cells and other regions showing attenuated cell density (Figure 2, B, C, F, G, and K). However, by 18 months, both $\mathrm{Hu}$ - and NeuN-labeled cells were more uniformly distributed through the graft/lesion site (Figure 2, I and L, and Supplemental Figure 2F). These findings, when taken together with the time course of neuron numbers in grafts, indicate that the period of subacute delayed cell loss may leave clusters of surviving NSCs or neuronal progenitor cells that gradually proliferate and distribute throughout the lesion site over a prolonged period of 1 year. Eventually, a mature neural environment forms in what would otherwise be an empty lesion site.

Grafted human glia mature gradually over 12 months. The expression of mature glial markers also occurred over a protracted, human-like time period (Figure 3). The glial progenitor marker vimentin was present at the earliest time point assessed, i.e., 1 month (Figure 3, A and B), and expression was continuously detected thereafter. The mature astrocytic cell marker GFAP was first detected within grafted cells 6 months after grafting, a late time point corresponding to human neural development (30) (Figure 3, C and D). At 18 months, we found that $48 \% \pm 1 \%$ of grafted, GFP-labeled NSCs expressed GFAP immunoreactivity. 

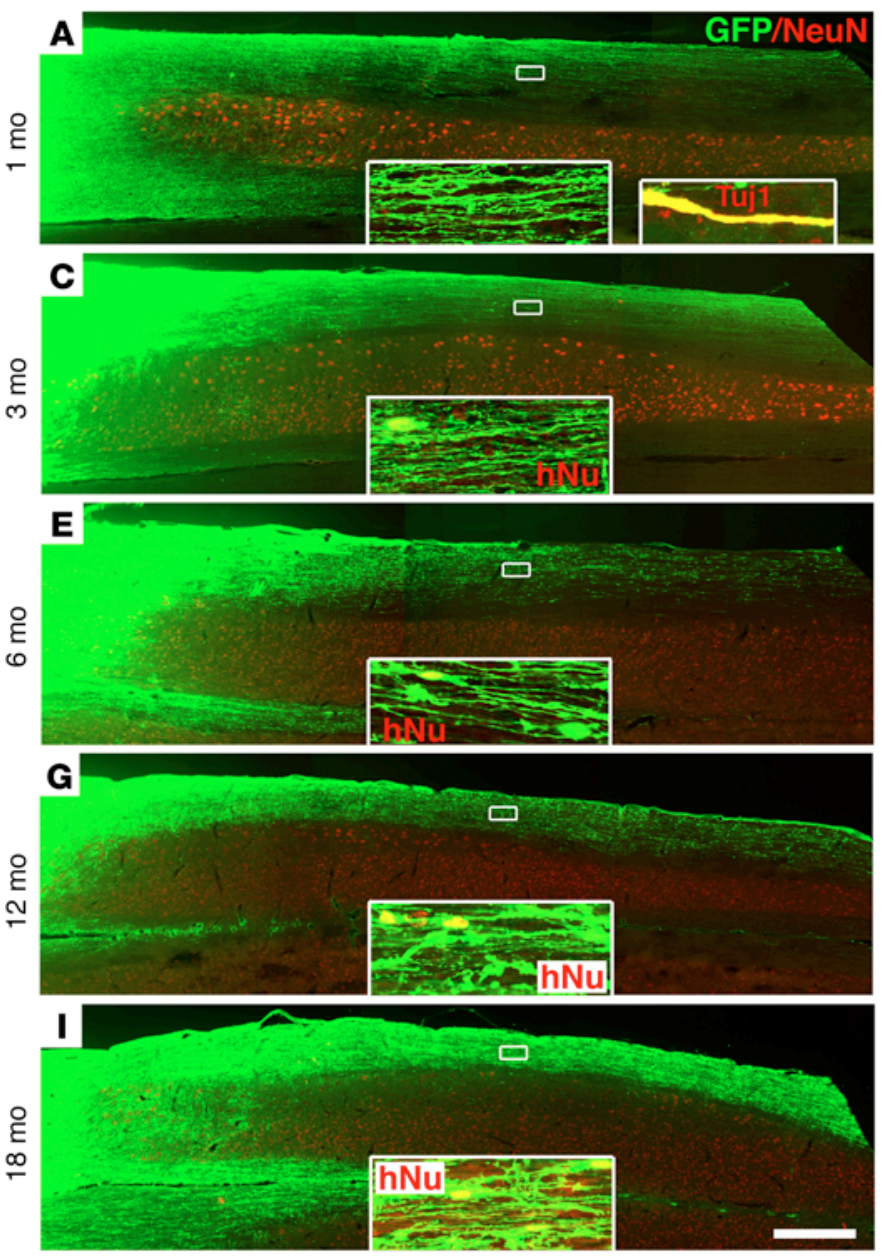

K

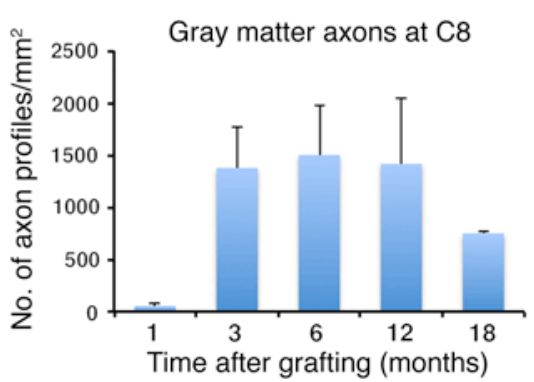

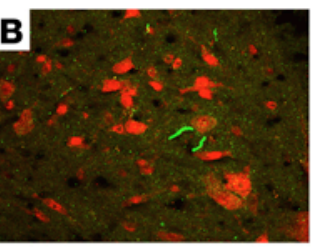
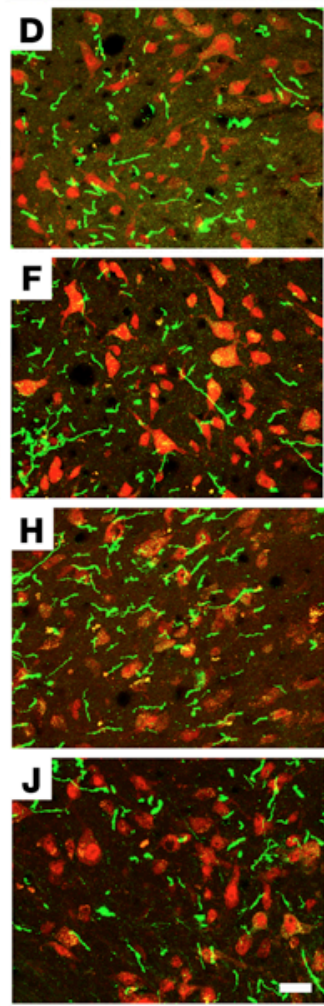

$\mathbf{L}$

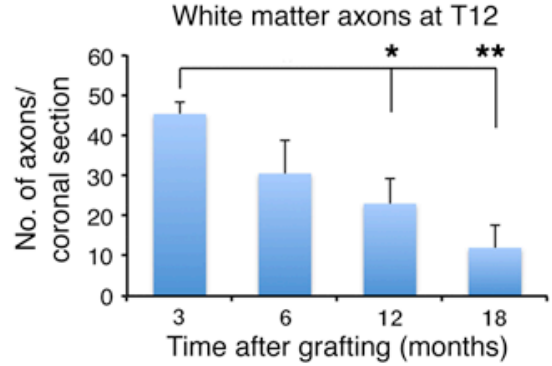

Figure 4. Axonal extension from H9-NSC grafts. (A and $\mathbf{B}$ ) Very large numbers of CFP-labeled human axons extended from the lesion site (far left side of the image) caudally after 1 month. (A) Horizontal section and (B) C8 coronal section. NeuN labels gray matter. Inset images show higher magnification of axons extending caudally in white matter, $3 \mathrm{~mm}$ caudal to the graft. The second inset in $\mathbf{A}$ shows colocalization of GFP with the axonal marker Tuj1, indicating the identity of many GFP processes as axons at 1 month. (C) Axons persisted 3 months after grafting, although glial migration was also evident at this point, as identified by the human nucleus marker hNu (inset; see also Figure 5). Because NSCs did not migrate into host gray matter, axon numbers over time could be quantified in gray matter. (D) A larger number of NSC-derived axons were evident in $\mathrm{C} 8$ gray matter at 3 months compared with those seen at 1 month, as quantified in $\mathbf{K}$. (E and $\mathbf{F}$ ) Six months after grafting, axons remained detectable in white and gray matter caudal to the lesion site. ( $\mathbf{G}$ and $\mathbf{H}$ ) By 12 months, a reduction in axon numbers caudal to the lesion site was observed, as quantified in $\mathbf{~ 8}$ gray matter in $\mathbf{K}$. (I and $\mathbf{J})$ Further attenuation of axon numbers was evident at 18 months. (K) Quantification of human NSC-derived axons in C8 gray matter Data represent the mean \pm SEM. $P=0.14$, by ANOVA for $1(n=3), 3(n=3), 6(n=5), 12$ $(n=3)$, and 18 months $(n=4)$. (L) Glial migration did not reach T12, thus GFP-labeled axon numbers could be reliably quantified over time at $\mathrm{T} 12$. A gradual reduction in axon numbers was evident. $P<0.05$, by ANOVA; ${ }^{*} P<0.05$ and ${ }^{* *} P<0.01$, by Fisher's exact post-hoc test for $1(n=3), 3(n=3), 6(n=5)$, $12(n=3)$, and 18 months $(n=3)$. Scale bars: $800 \mu \mathrm{m}$ (A, C, E, G, and I); $25 \mu \mathrm{m}$ (B, D, F, H, and J). Original magnification: $\times 1200$ for GFPTUJ1 and $\times 600$ for other inset images.
Oligodendrocyte maturation was the most prolonged of all the primary CNS cell types. The early oligodendroglial marker Oligo2 (31) was detected as early as 3 months after grafting (Figure 3, E and F). We used an adenomatous polyposis coli (APC) antibody to detect mature oligodendrocytes, because it labels the cell body (allowing clear quantification), and we used clone CC-1 of the APC antibody, because it is reportedly more specific for oligodendrocytes than polyclonal APC antibodies (32, 33). Notably, cells first expressed APC only 12 months after grafting (Figure 3, G and H). The proportion of all grafted GFP-labeled cells that coexpressed APC was $12 \% \pm 1 \%$ at 12 months and $11 \% \pm 1 \%$ at 18 months.

Having identified that the maturation of NSCs follows a human temporal course of development, we next sought to determine whether there was evidence of axonal pruning, another important aspect of developmental neurobiology.
Human axons project in large numbers over long distances, but their numbers are gradually reduced. As we reported previously (4, 5), human GFP-labeled axons emerged from grafts placed in the lesion site and extended rapidly and in large numbers over very long distances in the lesioned adult rat CNS (Figure 4). At the earliest time point sampled, 1 month after grafting, GFP-labeled axons had already extended 10 spinal segments caudally from the C5 lesion site to the T6 spinal cord, a distance of $27 \mathrm{~mm}$ (Figure 4, A and B, Supplemental Figure 5, A and B, and Supplemental Table 1). Axons also extended rostrally for a distance of $15 \mathrm{~mm}$ to the brainstem (Supplemental Figure 5, C and D, and Supplemental Table 1). The identity of these GFP-labeled processes as axons was confirmed by colocalization with the axonal marker $\beta$ III-tubulin (Tuj1) (Figure 4A, inset). Notably, while axons were present in both white and gray matter, many more axons were located in 

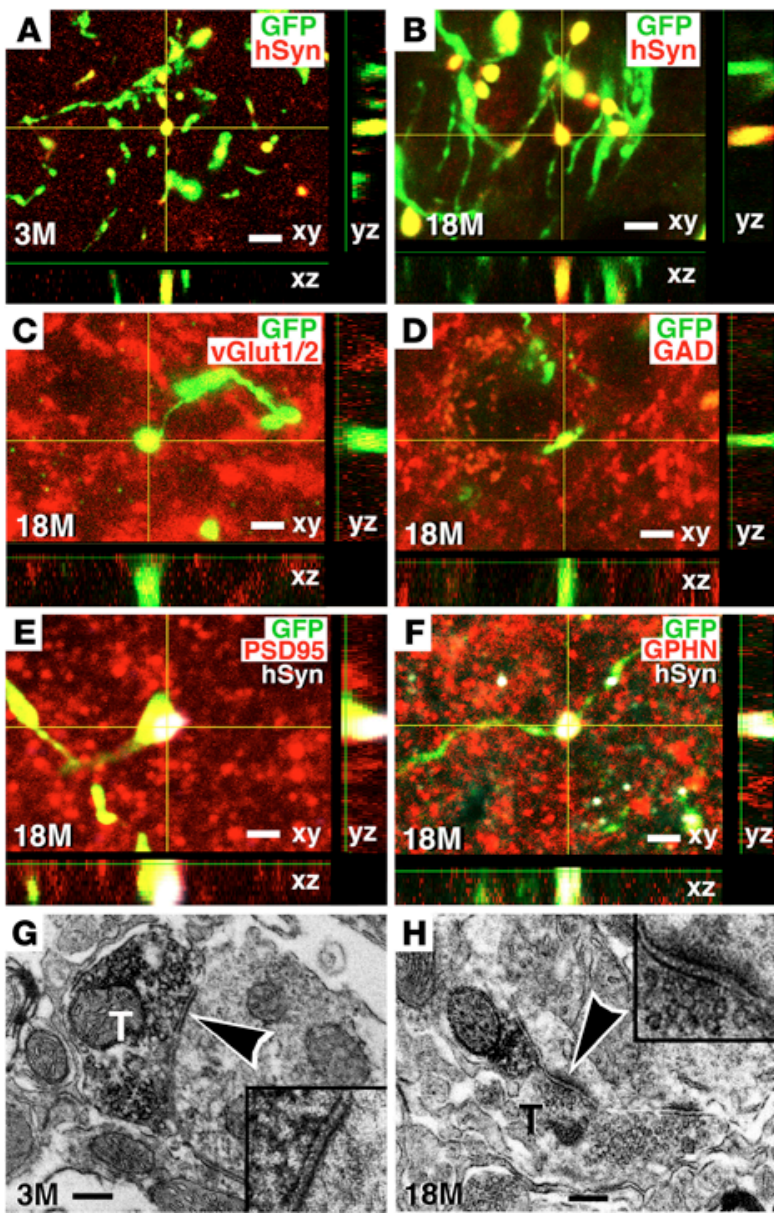

white matter than gray matter at the 1-month time point; branching into gray matter was relatively infrequent (Figure 4, A and B, and Supplemental Figure 5, A-D).

By 3 months after grafting, axons extended over even longer distances. Axons now reached the L 4 spinal cord, a distance of $57 \mathrm{~mm}$ or 21 spinal segments (Figure 4, C and D, Supplemental Figure 5, E-H, and Supplemental Table 1). Axons also extended longer distances in the rostral direction over a distance of 48 $\mathrm{mm}$, extending as far as the cortex and olfactory bulb, as previously reported $(4,5)$ (Supplemental Figure 5, I-N, and Supplemental Table 1). Moreover, the number of axons present in host gray matter (measured at the C8 spinal level, 3 spinal segments caudal to the lesion site) increased markedly (nearly 100-fold) from 1 to 3 months after grafting (Figure 4, B, D, and K). Notably, both the maximum distance over which axons extended and their density in host gray matter reached a maximum at 3 months (Figure 4, Supplemental Figure 5, E-N, and Supplemental Table 1). Neither maximum axon growth distance nor total axon numbers increased at the longer time points of 6,12 , and 18 months (Figure 4, E-J). Indeed, total axon numbers measured in white matter at the T12 spinal segment were maximal at 3 months and steadily declined by $75 \%$ at 18 months after grafting $(P<0.05$, by ANOVA) (Figure 4L and Supplemental Figure 6, A-D). Interestingly, axon density in host gray matter (measured at the C8 level) was also reduced at the 18 -month time point, but these reductions occurred later compared with white matter losses and were
Figure 5. Connectivity of human axons with host neurons. (A and B) GFP-immunoreactive human axons in host gray matter at C8 expressed hSyn at both 3 and 18 months after grafting, suggesting the formation of presynaptic elements. (C and $\mathbf{D})$ Some GFP-immunoreactive human axonal terminals expressed (C) vGlut1/2, suggesting the presence of excitatory synapses, or (D) GAD65/67, suggesting inhibitory synaptic terminals. (E and F) GFP and hSyn labeled presynaptic elements at human axonal terminals and were closely associated with individual excitatory and inhibitory postsynaptic proteins labeled with (E) PSD95 and (F) GPHN (all at C8 gray matter from subjects that survived 18 months). ( $\mathbf{G}$ and $\mathbf{H}$ ) Electron microscopy confirmed that DAB-labeled, GFP-expressing human axon terminals $(T)$ formed synapses (arrowheads) with host dendrites (see insets) 3 months and 18 months after grafting. Synapses were asymmetric and contained rounded vesicles, suggesting excitatory synapses in these views. Scale bars: $3 \mu \mathrm{m}$ (A, B, and D); $3.5 \mu \mathrm{m}$ (C); $3 \mu \mathrm{m}$ (E); $2.5 \mu \mathrm{m}$ (F); 550 $\mathrm{nm}(\mathbf{G}$ and $\mathbf{H})$. Original magnification in $\mathbf{G}$ and $\mathbf{H}$ insets: $\times 15,000$.

less extensive: axon numbers in host gray matter were approximately $50 \%$ of the numbers detected at 3 months (Figure $4 \mathrm{~K}$ ), although this late reduction was not significant $(P<0.1$, by ANOVA; $P=0.3$, by Fisher's exact post-hoc test comparing 12 months with 18 months after grafting). We measured axon numbers in host white matter at the T12 level, because we could clearly distinguish human axons from migrating human glia at this level (glia were never present at T12 except in 1 surviving 18 months that was excluded for axon number counting).

We further confirmed the identity of GFP-labeled processes as axons using the human-specific mature axonal marker neurofilament 70 (NF-70) (34). Expression of human NF-70 was clearly detected 12 and 18 months after grafting (Supplemental Figure $6, \mathrm{E}$ and $\mathrm{F}$ ), but not at 6 months or earlier time points, indicating that human axons, like human neurons, exhibit a protracted time course of maturation when grafted to sites of rodent SCI. We also observed colocalization of GFP-labeled axons with the early and late axonal marker Tuj1 (Figure 4A, inset).

Human axons in white matter frequently branched into host gray matter and formed axon terminal-like structures (Supplemental Figure 7A). Graft-derived human axons terminated in different zones of host gray matter, including dorsal, intermediate, and ventral host gray matter, with a preponderance of terminals in intermediate and ventral gray matter (Supplemental Figure 7, B-E).

Notably, human axons formed synapses with host rat neurons as early as 3 months after grafting (Figure $5 \mathrm{~A}$ ), despite the fact that neurons continued to mature for another 15 months. Synapses were also readily detected 18 months after grafting, indicating that synaptic projections onto host neurons could remain stable over time (Figure 5B). Some human axon terminals that projected onto host neurons were excitatory, as evidenced by the expression of vesicular glutamate transporter 1 and 2 (vGlut1/2) (Figure 5C). Other synapses expressed inhibitory transmitter markers (Figure 5D). In addition, human presynaptic elements were closely associated with the excitatory postsynaptic marker postsynaptic density 95 (PSD95) (Figure $5 \mathrm{E}$ ) (35) and the inhibitory postsynaptic protein gephyrin (GPHN) (Figure 5F) (36). Immunoelectron microscopy confirmed synapse formation between GFP-labeled, graft-derived human axons and host rat neurons at both 3 and 18 months after grafting (Figure 5, G and $\mathrm{H}$ ). 

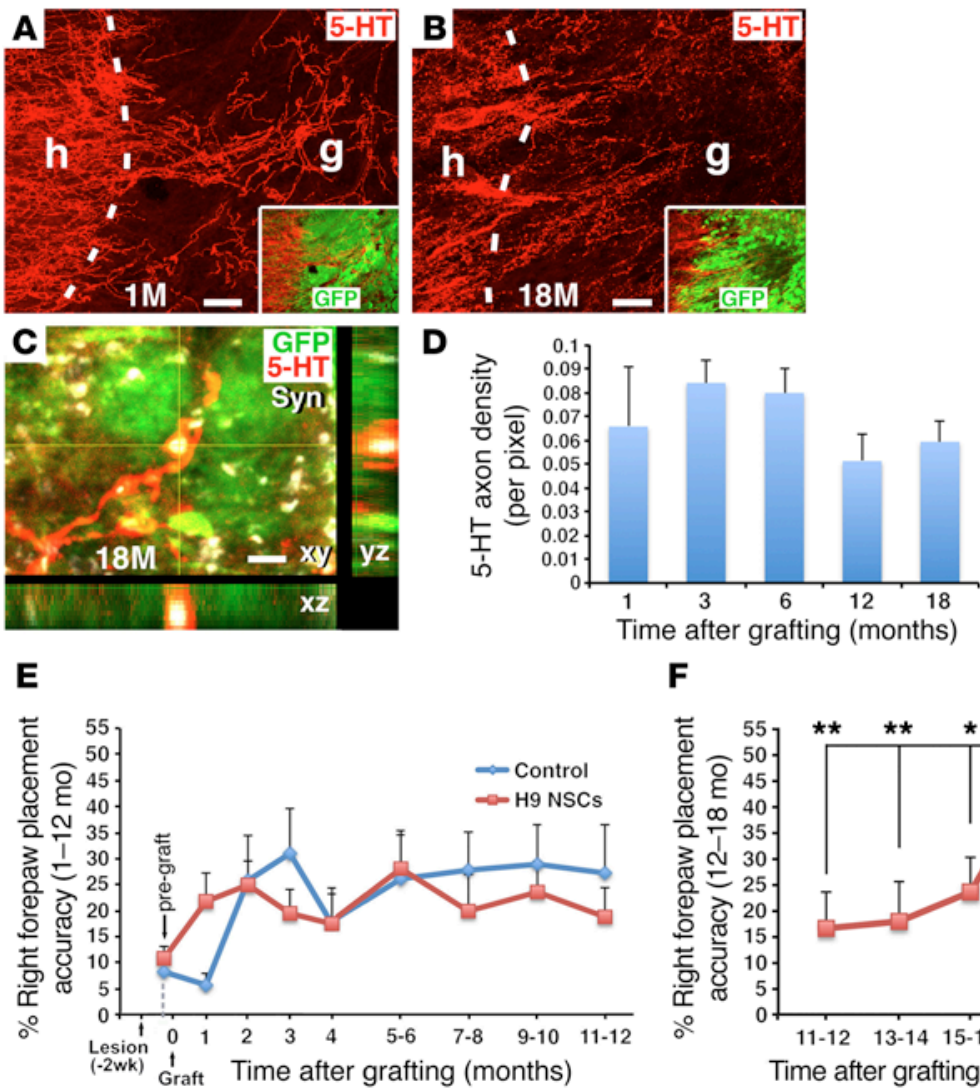

$\mathbf{F}$

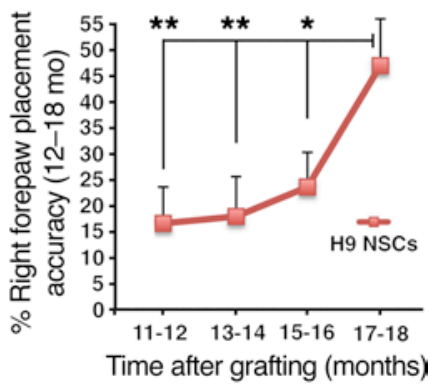

Figure 6. Host axonal regeneration and behavioral outcomes. (A and B) Host 5-HT-labeled serotonergic axons regenerated into GFP-expressing human NSC grafts in a C5 hemisection lesion site 1 and 18 months after grafting. Dashed lines indicate the host/graft $(\mathrm{h} / \mathrm{g})$ interface. (C) Higher-magnification image shows 5-HT-labeled host axons regenerating into a GFP-expressing human NSC graft. The 5-HT-labeled structures colocalized with Syn in a Z-stack image, 18 months after grafting. (D) 5-HT quantification reveals that host serotonergic penetration of grafts was stable over time ( $P=0.7$, by ANOVA), for 1 $(n=3), 3(n=3), 6(n=5), 12(n=3)$, and 18 months $(n=4)$. (E) Forepaw placement accuracy on a behavioral grid-walking task was measured monthly for the first 4 months and bimonthly thereafter. Following an initial period of modest recovery from 0 to 2 months after lesioning, functional performance thereafter was stable in both grafted $(n=7)$ and control lesion $(n=5)$ groups and did not improve from 1 to 12 months $(P=0.62$, by 2-way repeated-measures ANOVA comparing graft and lesion-only groups from 1 to 12 months). The control group was perfused at a preplanned anatomical endpoint of 12 months. (F) Functional testing in NSC-grafted animals continuing beyond 12 months $(n=4)$ revealed a significant, 2.7-fold late recovery of function compared with their own stable functional baseline at 12 months. ${ }^{*} P<0.02$ and ${ }^{*} P<0.05$, by post-hoc Fisher's exact test comparing performance at 18 months at the indicated time points. Scale bars: $58 \mu \mathrm{m}$ (A and B); $8 \mu \mathrm{m}$ (C)
Host axons stably penetrate human NSC grafts. We examined host serotonergic axon penetration of human NSC grafts, as serotonergic systems play important modulatory roles in motor function $(37,38)$. We found that serotonergic axons penetrated grafts as early as 1 month after injury, and the distance of penetration and density of serotonergic axons in grafts remained stable up to 18 months after grafting (Figure 6, A, B, and D). Thus, in contrast to axons growing out of NSC grafts, adult axons regenerating into NSC grafts were stable and were not reduced in number over time. Serotonin (5-HT) somata were not present in human NSC grafts, indicating that serotonergic axons in grafts were host derived. In addition, regenerated serotonergic host axons expressed synaptophysin (Syn) (Figure 6C), suggesting potential synaptic connectivity with grafted neurons.

Function. Because human NSCs gradually mature over 18 months, prolonged time periods may be required to observe the functional effects of these cells compared with grafts of rodent NSCs, which mature far more rapidly and can support functional improvement within 3 months $(4,8,39)$. Complete C5 right hemisection lesions cause lasting deficits in forelimb use, which can be assessed by measuring forepaw placement as animals walk on an irregular grid. Over a 1- to 12-month period, the functional performance of both grafted and lesioned control groups was identical ( $P=0.62$, by 2 -way repeated-measures ANOVA) (Figure 6E). Moreover, while forepaw placement improved slightly from month 0 after lesioning to month 2 in all lesioned animals, as expected, function thereafter did not improve further, up to 1 year (ANOVA $P=0.87$ for the grafted group from months 2 through 12 after lesioning). However, beginning at month 12, performance in the grafted group improved progressively from $17 \%$ accuracy in right forepaw placement to $47 \%$ accuracy by month 18 , a 2.7 -fold improvement that was statistically significant $(P<0.01$, by ANOVA comparing all time points in the grafted group between 12 and 18 months; $P<0.01$, by Fisher's exact post-hoc test comparing month 12 with month 18 ) (Figure 6F). The control group animals were sacrificed at month 12 as a preplanned study anatomical endpoint. However, performance of all lesioned animals plateaued 2 months after injury (Figure 6E) and would not have been expected to improve spontaneously after month 12 (40). Moreover, human clinical trials would be designed to detect late, within-subjects improvement from a chronic, stable baseline score, as in this study, because patients typically show little or no improvement in function at chronic post-injury time points (41-43).

Glial migration from human grafts into host white matter. We observed a slow and persistent migration of human astrocytes from the graft site into the host spinal cord over 18 months (Figure 7 and Supplemental Figure 8). Migrating GFP-immunoreactive cells were colabeled for the human cell-specific nuclear marker hNu and for the mature astrocyte marker GFAP (Figure 7), but did not colocalize with either neuronal (NeuN, Tuj1), oligodendroglial (APC, Oligo2, NG2) (Supplemental Figure 8, $\mathrm{H}$ and I), or NSC (nestin) markers (data not shown). Astrocytes migrated from the lesion site at a rate of 2 to $3 \mathrm{~mm}$ per month. One month after grafting, cell migration from the lesion site was not evident, but we observed migration into host white matter by 3 months after grafting for a distance of 5 to $6 \mathrm{~mm}$ (Figure 7, A and B, and Supplemental Table 1). This increased to 

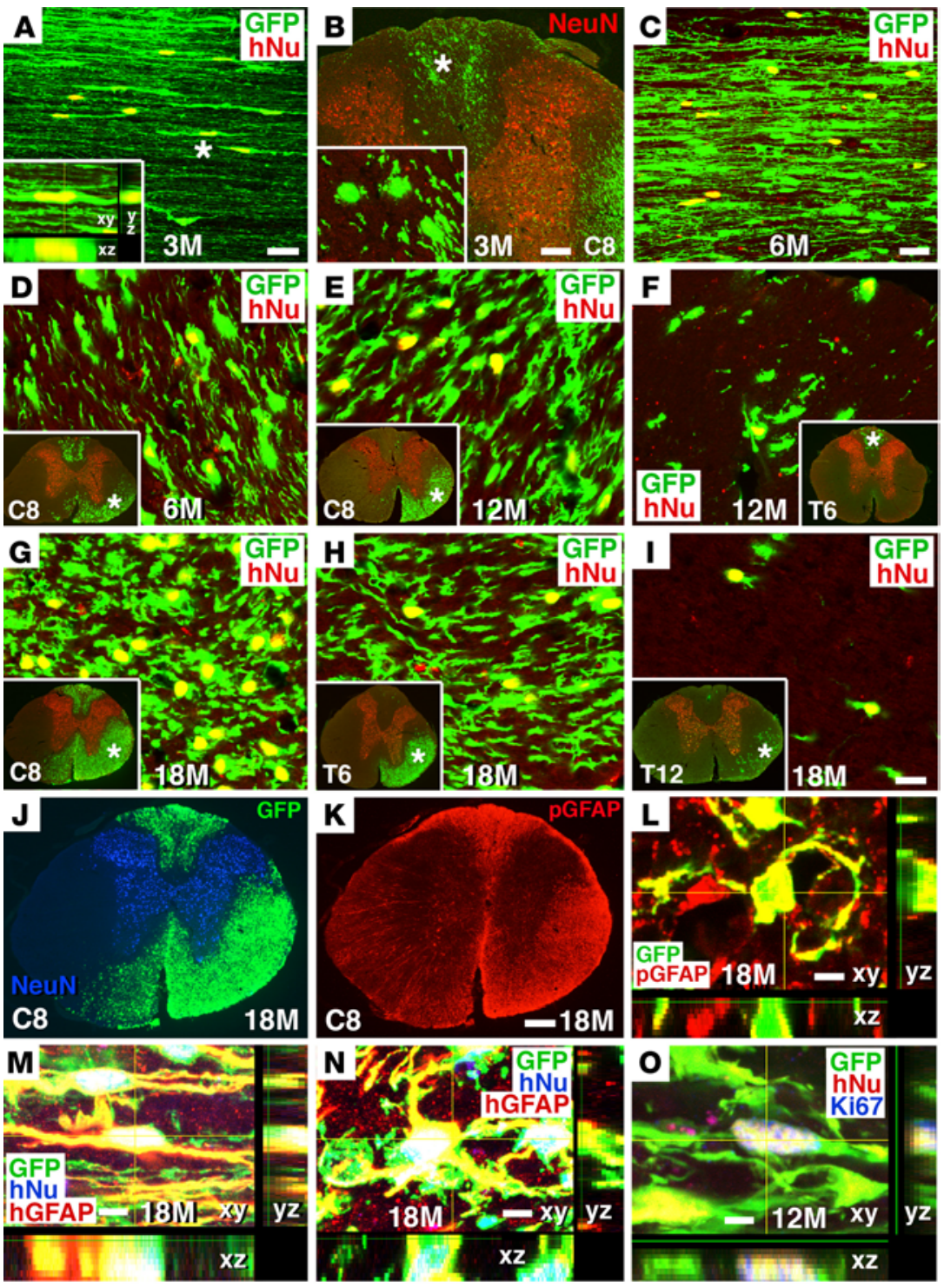

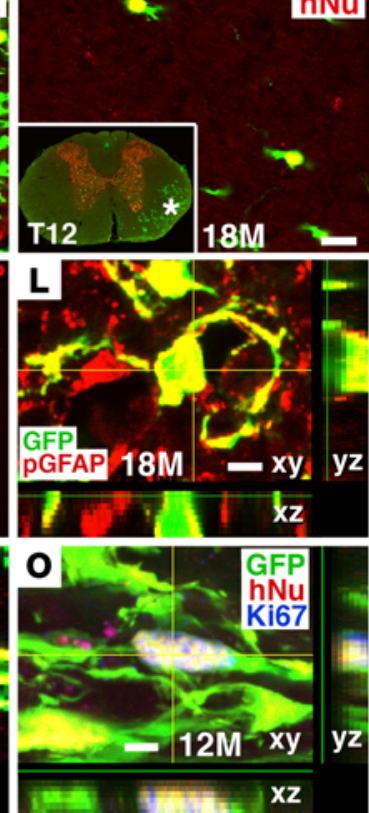

Figure 7. Human glial migration from graft site into host white matter. (A and B) Human NSCs migrated out of the graft into host white matter, beginning 3 months after grafting, as indicated by colocalization of GFP with the human-specific nuclear marker hNu. (A) Horizontal section $3 \mathrm{~mm}$ caudal to the graft (inset is a Z-stack image); (B) coronal section at C8 (inset is a higher-magnification image from the dorsal column region, indicated by an asterisk). (C and D) More extensive human NSC migration was evident 6 months after grafting at the same level as in $\mathbf{A}, 3 \mathrm{~mm}$ caudal to the graft. (E and $\mathbf{F}$ ) Human cell migration at $\mathrm{C} 8$ and $\mathrm{T} 6$ after 12 months; insets show regions of sampling, colabeled for NeuN. (G-I) Human cell migration at C8, T6, and T12 at 18 months. (J and $\mathbf{K}$ ) Migrated human cells (GFP+) colocalized with pan-GFAP (pGFAP); c8 coronal section 18 months after grafting. (L) Confocal Z-stack image showing colocalization of GFP and pGFAP at C8. ( $M$ and N) Z-stack images showing colocalization of GFP+ and $\mathrm{hNu}^{+}$human cells with the human-specific astroglial marker hGFAP, 18 months after grafting. (M) Horizontal section $3 \mathrm{~mm}$ caudal to the graft; ( $\mathbf{N}$ ) coronal section at C8. (0) Z-stack image showing GFP and hNu coexpress ing the cell proliferation marker Ki67, 12 months after grafting in a C7 horizontal section. Scale bars: $28 \mu \mathrm{m}$ (A and C); $100 \mu \mathrm{m}$ (B); $18 \mu \mathrm{m}$ (D-I); $260 \mu \mathrm{m}$ (J and K); $3 \mu \mathrm{m}(\mathbf{L}) ; 7 \mu \mathrm{m}(\mathbf{M}) ; 5 \mu \mathrm{m}$ (N and $\mathbf{0})$. Original magnification of insets: $\times 1200(\mathbf{A}) ; \times 400(B) ; \times 40($ D-I).
12 to $15 \mathrm{~mm}$ at 6 months (Figure 7, C and D, and Supplemental Figure 8, A and B); 24 to $36 \mathrm{~mm}$ at 12 months (Figure 7, E and F, Supplemental Figure 8, C and D, and Supplemental Table 1); and up to $45 \mathrm{~mm}$ at 18 months (Figure 7, G-I, Supplemental Figure $8, \mathrm{E}-\mathrm{G}$, and Supplemental Table 1). Migration occurred in both rostral and caudal directions (Figure 7 and Supplemental Figure 8), but only in white matter; no human astrocytes were detected in host gray matter (Figure 7 and Supplemental Figure 8). Cells divided as they migrated, as evidenced by occasional labeling for Ki67 in these migrating cells (Figure 7O). In host white matter, we quantified the proportion of human cells that expressed Ki67 at spinal level C8, three segments below the lesion site, and this proportion gradually declined over time: $4.6 \% \pm 0.7 \%$ at 6 months; $3.4 \% \pm 2.8 \%$ at 12 months; and $0.34 \%$ $\pm 0.21 \%$ at 18 months. Most migrating human astrocytes had typical bipolar astrocyte morphology, with long processes that were oriented in a rostrocaudal direction (Figure 7 and Supplemental Figure 9A). Human astrocytic processes were closely associated with vascular endothelium, labeled by REC1, and surrounded blood vessels (Supplemental Figure 9B), suggesting the formation of a blood-brain barrier (44).

Ectopic cell collections were rare. It has been reported that grafts of NSCs can form ectopic cell deposits remote from the site of grafting $(45,46)$. In this experiment, remote cell deposits occurred in 2 of 18 animals (11\%) (Supplemental Figure 9, C-F), and local extra-pial cell spreading was confined to 1 to 2 spinal segments from the graft site in 5 of 18 animals (28\%). None of these extra-axial cell collections resulted in detectable compression or damage to underlying host neural tissue, even after 18 months, and labeling for astrocyte and microglial/macrophage markers failed to reveal evidence of a host response to the overlying human cells (Supplemental Figure 9, C and D). One remote ectopic cell deposit was present on the dorsal spinal cord surface at T12 cord in an animal surviving 12 months (Supplemental Figure 9C), and a second remote cell deposit was present in the dorsal medulla of an animal surviving 18 months (Supplemental Figure 9, E and F). In neither case was there secondary compression of the underlying host spinal cord or brainstem after this prolonged time period. 


\section{Discussion}

We report here what to our knowledge is the longest survival period to date - 18 months - of NSCs implanted into sites of neurotrauma; to our surprise, we found evidence of continued stem cell maturation throughout this period. Neurons continued to hypertrophy and increase NeuN expression; mature astrocyte markers first appeared 6 months after grafting; and mature oligodendrocyte markers were not evident until 12 months after grafting. These findings clearly indicate that intrinsic human maturation "programs" are retained by NSCs placed in sites of neurotrauma and that neither the adult environment, the injured environment, nor the rodent environment alters this prolonged developmental time course. Moreover, recovery of forelimb function is supported by human NSC grafts, but only after mature cell markers of both neuronal and glial lineages are expressed.

In another parallel with normal human development, axons extended early and profusely from neural implants, and their numbers were pruned over 1 year to approximately half their original numbers in gray matter and to one-quarter their original numbers in white matter (Figure 4). These reductions are consistent with observations in neural development (47). This reduction in axon numbers observed in our study is not likely to be attributable to a downregulation in expression of the reporter gene GFP, since it is expressed under the ubiquitin promoter. Developmental cell death is another feature of normal nervous system development (48), and our grafted human NSCs exhibited a reduction of approximately $40 \%$ in total cell numbers from 3 months to 6 months after grafting. Interestingly, neuron numbers recovered at 12 and 18 months, points at which neurons also became more evenly distributed throughout the graft in the lesion site (Figure 2).

Human NSC-derived axons grew into both caudal and rostral host regions, including some ectopic regions such as the cerebral cortex and olfactory bulb. While ectopic axonal growth could result in adverse functional effects, the number of axons reaching these ectopic targets was very small, and gross functional deficits were not noted in these animals. In addition, axons may not reach these distant targets in the substantially longer human nervous system. Future long-term studies will be required to fully characterize the safety of this approach. Notably, neurons did not migrate from the lesion site, but astrocytes slowly migrated into host white matter at a rate of 2 to $3 \mathrm{~mm}$ per month. By 12 to 18 months, human glia represented a substantial proportion of astrocytes in host white matter both caudal and rostral to the lesion site (Figure 7 and Supplemental Figure 8), raising potential safety concerns. However, adverse effects of this glial outgrowth were not evident as either tumor formation or deterioration in forelimb function over time. Previously, Goldman and colleagues reported long-term safety and efficacy after administration of human glial progenitor cells in rodent demyelinating disease models (15), and Zhang and colleagues reported the functional integration of human-derived astrocytes into intact rodent spinal cord, including investment of astrocytic processes around host neurons and extension of end feet around blood vessels (44). Similarly, ectopic clusters of cells that have been previously reported $(45,46)$ with our grafting technique were only rarely observed at prolonged time points ( 2 of 12 animals surviving 6 months or longer) and did not compress the spinal cord. Nonetheless, for optimization of safety, grafting techniques can be used that minimize graft cell leakage into the cerebrospinal fluid after implantation in closed lesion sites; such measures eliminate ectopic cell collections (8).

Overall, our NSC grafts generated from the human ESC line H9 preliminarily showed acceptable safety outcomes over this prolonged experiment. Grafts did not expand over time, and cell division steadily declined over time, with a complete absence of dividing cell markers in 2 of the 4 animals examined at 18 months. This is useful long-term safety data for potential future human clinical trials.

The observation that NSC grafts continue to mature for at least 18 months and support late functional recovery has important implications for the design of human clinical trials. We propose that preplanned clinical outcome measures will need to be focused on equally long time points after grafting. Reliance on earlier time points for primary outcome measures could lead to a misleadingly negative interpretation of outcomes; instead, trial design will need to account for the prolonged developmental biology of NSCs.

\section{Methods}

Human ESC-derived NSCs. Generation of the human H9 ESC line and differentiation of NSCs from these ESCs have been described previously $(19,20)$. Briefly, human H9 ESCs were derived from the inner cell mass of a blastocyst-stage embryo with a normal female XX karyotype; these cells expressed primate ESC markers but not other early lineage markers (19). H9 ESCs were induced to differentiate into NSCs by embryonic body and neural tube-like structure formation in response to the presence of FGF-2. Differentiated NSCs were isolated by selective enzymatic digestion and further purified on the basis of differential adhesion (20). The H9 ESC-derived NSCs (H9-NSCs) expressed the NSC marker nestin and were able to differentiate into neurons, astrocytes, and oligodendrocytes in vitro following withdrawal of FGF-2. The H9-NSC line was obtained from Aruna Biomedical and Invitrogen (Thermo Fisher Scientific). We purchased H9-NSCs from Invitrogen (catalog N7800-100) and cultured H9-NSCs as a monolayer on a CELLstart coated flask (Thermo Fisher Scientific) in KnockOut DMEM/F-12 with supplement and FGF-2 and EGF. To track the transplanted human NSCs in vivo, we transduced proliferating H9-NSCs with lentiviral vectors expressing GFP (49). Approximately 95\% of the H9-NSCs expressed GFP.

In vivo studies. A total of 26 adult female athymic, T cell-deficient nude rats (Harlan Laboratories) weighing 180-200 g were used in this study. The animals were deeply anesthetized using a combination (2 $\mathrm{ml} / \mathrm{kg})$ of ketamine $(25 \mathrm{mg} / \mathrm{ml})$, xylazine $(1.3 \mathrm{mg} / \mathrm{ml})$, and acepromazine $(0.25 \mathrm{mg} / \mathrm{ml})$ under aseptic conditions. All 26 rats received a right C5 hemisection as described previously $(5,50)$. Briefly, following C5 dorsal laminectomy, the dura was cut longitudinally and retracted. A 1.5-mm-long block of the right spinal cord was excised using a combination of iridectomy scissors and microaspiration, with visual verification to ensure complete transection ventrally, medially, and laterally. The lesion was closed, and the subjects were treated with the antibiotics ampicillin (3-5 mg/kg/day) via a s.c. daily injection for 1 to 2 weeks and amoxicillin and sulfamethoxazole in the drinking water $(0.5 \mathrm{mg} /$ $\mathrm{ml}$ ) continuously until sacrificed.

Two subjects died one day after C5 hemisection, and one animal was perfused one week after C5 hemisection due to systemic illness (prior to grafting); none of the animals died after stem cell grafting. 
The remaining 23 subjects were randomly divided into human NSC graft $(n=18)$ or control $(n=5)$ groups. Two weeks later, cultured human H9-NSCs were trypsinized, washed with PBS, and resuspended at a concentration of 250,000 cells/ $\mu$ l. Cells were mixed into a fibrin matrix containing a growth factor cocktail to promote survival and retention in the lesion site, as previously described $(4,5)$. The fibrin and growth cocktail alone (lacking NSCs, in controls) or human NSCs (5 $\mu \mathrm{l})$ in the fibrin and growth factor cocktail were microinjected into the subacute lesion cavity using a pulled glass micropipette with an inner diameter of $40 \mu \mathrm{m}$, connected to a Picospritzer II (General Valve). Cells were injected into 6 sites encompassing the lesioned hemicord: 2 sideby-side injections were made at the center of the lesion cavity, spaced $0.5 \mathrm{~mm}$ apart (in the mediolateral plane); 2 side-by-side injections were made $0.5 \mathrm{~mm}$ rostral to the center of the lesion site, spaced $0.5 \mathrm{~mm}$ apart (in the mediolateral plane); and 2 side-by-side injections were made $0.5 \mathrm{~mm}$ caudal to the center of the lesion site, spaced $0.5 \mathrm{~mm}$ apart (in the mediolateral plane). Approximately $1 \mu \mathrm{l}$ cell matrix per site was injected, for a total volume of approximately 5 to $6 \mu$ l. Injections were stopped if reflux occurred. After human NSC transplantation or vehicle injection, the animals were sacrificed at different time points as a time-course study of NSC survival, differentiation, and maturation: 1 month $(n=3) ; 3$ months $(n=3) ; 6$ months $(n=5) ; 12$ months ( $n=3$ for the NSC group and $n=5$ for the controls); and 18 months $(n=4)$.

Functional testing. Forepaw placement was examined on a gridwalk task. Footfalls were measured in 5-minute test sessions recorded on video as animals walked on a $38 \mathrm{~cm}^{2}$ plastic-coated wire mesh field containing $3-\mathrm{cm}^{2}$ openings. Each forepaw was scored for the total number of steps and the total number of missteps. Step and misstep numbers were added to obtain the total number of placements. By convention, the right forepaw correct placement was calculated by dividing the number of steps by the total number of placements (steps plus missteps) $(5,51)$. Functional testing was performed for all animals surviving to 12 months (7 grafted animals and 5 controls) and continued for an additional 6 months for 4 grafted animals. The lesioned control animals did not continue functional testing beyond 12 months, because they had reached a preplanned anatomical endpoint for comparison with grafted animals. Initial testing was performed 1.5 weeks after C 5 hemisection to obtain post-lesion scores, and then testing was performed monthly for 4 months and at least bimonthly thereafter $(5,51,52)$.

Anatomical analysis. Rats were transcardially perfused with $4 \%$ paraformaldehyde in $0.1 \mathrm{M}$ phosphate buffer $(\mathrm{pH}=7.4)$ and post-fixed overnight. Spinal cords and brains were dissected out and transferred to $30 \%$ sucrose for 72 hours. A $12-\mathrm{mm}$ length of spinal cord containing the NSC graft in the middle was entirely sectioned horizontally through its ventral-to-dorsal axis on a cryostat set at $30-\mu \mathrm{m}$ thickness, and the horizontal sections were serially collected into 24 -well plates for immunohistochemical analysis. In addition, the remaining spinal cord was blocked into 1- to 2-mm segments at C2, C8, T6, T12, and L4 spinal cord levels for coronal sections, and the whole right hemisphere of the brain was sectioned sagittally and collected into 24 -well plates. Free-floating spinal cord and brain sections were processed for immunohistochemical analysis with the following primary antibodies: (a) GFP rabbit polyclonal antibody (A6455; Invitrogen, Thermo Fisher Scientific; 1:3,000) and GFP chicken polyclonal antibody (ab13970; Abcam; 1:3,000) for GFP labeling to assess grafted cell survival, differentiation, and processes outgrowth; (b) hNu mouse monoclonal antibody (MAB1281, clone 235-1; EMD Millipore; 1:200) for human nuclei labeling to assess grafted human cell bodies; (c) rabbit polyclonal antibody (ab16667, Abcam; 1:500) for Ki67 labeling to assess proliferating cells; d) mouse monoclonal antibody (10C2; Gene Tex; 1:500) for neural cell markers, including human-specific nestin; goat polyclonal DCX antibody (sc-8066; Santa Cruz Biotechnology Inc.; 1:1,000) for immature neurons; Hu human antibody for immature and mature neurons (1:1,000; a gift of Robert Darnel, Rockefeller University, New York, New York, USA) (25); Tuj1 mouse monoclonal antibody (MO15013; Neuromics; 1:200) for immature and mature neurons; NeuN mouse monoclonal antibody (ab104224, clone 1B7; Abcam; 1:1,000) for mature neuronal nuclei; goat polyclonal choline acetyltransferase (ChAT) antibody (AB144P; EMD Millipore; 1:200) for mature spinal motor neurons; human-specific NF-70 mouse monoclonal antibody (MAB5294, clone DP5 2.7.3; EMD Millipore; 1:1,500) to label human axons; 5-HT rabbit polyclonal antibody (20080; ImmunoStar; 1:10,000) for mature raphespinal neurons and axons; mouse monoclonal Syn antibody (MAB5258, clone SY38; EMD Millipore; 1:1,000) for presynaptic proteins expressed on both human and rodent synapses; hSyn mouse monoclonal antibody (NBP1-19222, clone EP10, Novus; 1:1,500) for human presynaptic proteins; vGlut1/2 mouse monoclonal antibody (MAB5502, clone 3C10.2, MAB5504, clone 8G9.2, Chemicon; 1:1,000) to label glutamatergic terminals; glutamic acid decarboxylase 65 or 67 (GAD65/67) to label GABAergic neurons/terminals (GAD65 goat polyclonal antibody, catalog AF2247, from R\&D Systems, or GAD67 mouse monoclonal antibody, catalog MAB5406, clone 1G10.2, from EMD Millipore; 1:1,000); PSD-95 rabbit polyclonal antibody (ab18258, Abcam; 1:500) to label postsynapse density; GPHN rabbit polyclonal antibody (ab32206, Abcam; 1:1,000) to label postsynapse density; GFAP mouse monoclonal antibody (MAB360, clone GA5, EMDMillipore; 1:1,500) to label astrocytes; human-specific GFAP (hGFAP) rabbit polyclonal antibody (TA302094; OriGene; 1:1,000); APC mouse monoclonal antibody (OP80, clone CC-1; Oncogene; 1:800) to label oligodendrocytes; Oligo2 rabbit polyclonal antibody (18953; IBL; 1:200) to label immature and mature oligodendrocytes; vimentin mouse monoclonal antibody (MAB3400, clone V9; EMD Millipore; 1:80) to label immature oligodendrocytes; NG2 rabbit polyclonal antibody (AB5320; EMD Millipore; 1:400) to label immature oligodendrocytes; CNPase mouse monoclonal antibody (NE1020, clone SMI-91; EMD Millipore; 1:200) to label immature oligodendrocytes; PDGFR- $\alpha$ goat polyclonal antibody (sc-31178; Santa Cruz Biotechnology Inc.; 1:500) to label immature oligodendrocytes; GalC mouse monoclonal antibody (MAB342, clone mGalC; EMD Millipore; 1:100) to label immature oligodendrocytes; A2B5 mouse monoclonal antibody (ab-53521, clone 105; Abcam; 1:100) to label glial progenitors; myelin basic protein (MBP) rabbit polyclonal antibody (AB980; EMD Millipore; 1:250) to label myelin; and nestin mouse monoclonal antibody (556309, clone rat 401; BD Biosciences; 1:200) to label NSCs. Sections were incubated overnight at $4^{\circ} \mathrm{C}$ for primary antibodies individually or in combination and then incubated with Alexa 488-, 594-, or 647-conjugated goat or donkey secondary antibodies (Invitrogen, Thermo Fisher Scientific; 1:500) for 2.5 hours at room temperature. The sections were then washed, mounted on uncoated slides, and coverslipped with Fluoromount-G (SouthernBiotech).

The NSC graft area was measured using Image J software (NIH) on images of fixed box size at 1,600 $\times 1,200$ pixels and $\times 20$ magnification, using every sixth horizontal section that contained GFPexpressing graft. The pixel value was converted into $\mathrm{mm}^{2}$ to obtain the average graft area in $\mathrm{mm}^{2}$ per section. The ratio of $\mathrm{Hu}^{+}$neurons in 
the NSC graft was determined by counting individual cells labeled for $\mathrm{Hu}$ within a fixed box size of $1,024 \times 1,024$ pixels in confocal images at $\times 600$ magnification within the graft, divided by the total number of cells per sample box labeled with the human nuclear antigen $\mathrm{hNu}$. Two randomly selected fields from the graft epicenter in two randomly selected horizontal sections were counted in each subject for each label. Exposure times were adjusted such that the brightest cell in each image was just below saturation. For each animal, 2 sections were quantified, with 1 grayscale image from both the rostral and caudal halves of the graft, for a total of 4 images per animal. For quantification of the size of $\mathrm{Hu}^{+}$and $\mathrm{NeuN}^{+}$neurons, images were converted to 8-bit and cropped to an $800 \times 800$ pixel region in the center of the original 1,024 $\times 1,024$ pixel images. The dimensions of the cropped images were $155 \times 155 \mu \mathrm{m}$. Each $\mathrm{Hu}^{+}$or $\mathrm{NeuN}^{+}$cell was outlined with the freehand tool and measured for area. Cells that extended to the borders of the image were not counted. The areas of $\mathrm{Hu}^{+}$or $\mathrm{NeuN}^{+}$cells in each animal were averaged and converted from pixels to square micrometers to produce the average $\mathrm{Hu}$ or NeuN size value.

The peak Hu and NeuN fluorescence intensity of grafted cells was quantified using ImageJ. A $\times 400$ image was captured from horizontal sections in areas containing both the rostral and caudal halves of each graft and in which the brightest, most homogenous distribution of $\mathrm{NeuN}^{+}$cells could be found. An exposure time of $141 \mathrm{~ms}$ was used for all images. Bright, relatively large, and in-focus cell perimeters were outlined freehand, and an adjacent background area was selected for each cell. Corrected total cell fluorescence was calculated by subtracting the product of the area of a cell and the mean gray value of its respective background selection from the integrated density of the cell. A total of 20 cells from 2 sections were analyzed for each subject.

For estimation of the total number of $\mathrm{Hu}^{+}$and $\mathrm{NeuN}^{+}$neurons, 2 sections per subject were imaged at $\times 200$ using a Keyence BZ-X710 automated fluorescence microscope. Each graft region was imaged in its entirety by stitching together full-focused, $960 \times 720$ pixel images captured automatically on the basis of 4 edge points representing the furthest extent of the graft (defined by dense GFP signal) in each $x-y$ direction. At each edge point, the top and bottom planes of the $Z$-stack to be captured were set. A slice interval of $1.5 \mu \mathrm{m}$ was used for all images. The exposure was set to a point at which the brightest $\mathrm{Hu}^{+}$or $\mathrm{NeuN}^{+}$ cell in the graft was just below saturation. Images were captured in both 488-nm (GFP) and 594-nm (Hu or NeuN) channels during each run. Images were then analyzed using Fiji software (ImageJ). Graft regions were outlined with the Polygon Selection tool. The graft border was defined as the edge of continuous, dense, and homogenous GFP signal. Ectopic $\mathrm{GFP}^{+}$regions were not included as part of the graft. The area outside of the graft was then cleared from the image, and the red $\mathrm{Hu}$ or NeuN channel was isolated for analysis. A series of repeating $400 \times 400$ pixel regions of interest (ROIs) was then superimposed on the image, with 400 pixels between ROIs on the $x$ axis, and 200 pixels on the $y$ axis, resulting in one-third sampling of the graft area in each image. Within each $\mathrm{ROI}, \mathrm{Hu}^{+}$or $\mathrm{NeuN}^{+}$cells with visible nucleoli contained entirely within the cropped graft were counted. Cells lying on the border of the ROI were only counted on the top and right edges. All ROIs containing any amount of graft area were quantified. The graft area was measured by setting the brightness threshold between 1 and 255 , eliminating all pixels with a brightness value of 0 (the cropped out area of the original image), and using the Measure function. For each image, the number of $\mathrm{Hu}^{+}$or $\mathrm{NeuN}^{+}$cells was divided by the area of the graft in that image and averaged with the values for the other image from that subject to obtain the average $\mathrm{Hu}$ or NeuN density as number/square pixel. This density was then converted to the number of neurons $/ \mathrm{mm}^{2}$ and multiplied by the average total graft area and the total number of sections per subject to obtain the estimated total number of neurons/graft.

To estimate the total number of human cells in grafts (labeled for $\mathrm{hNu}$ ), we calculated the ratio of $\mathrm{Hu}$-labeled neurons to total $\mathrm{hNu}$-labeled human nuclei in thin $(1-\mu \mathrm{m})$ double-labeled $(\mathrm{hNu} /$ $\mathrm{Hu})$ sections at $\times 600$ magnification. Ratios were quantified from 2 anatomical regions per graft, and regions were pseudo-randomly selected by StereoInvestigator software. The total number of $\mathrm{Hu}-$ labeled cells per graft was divided by the ratio of $\mathrm{Hu} / \mathrm{hNu}$ as follows: total number of human cells $=$ (total number of Hu neurons) $/$ (ratio of $\mathrm{Hu} / \mathrm{hNu}$ ). This procedure was used because individual hNulabeled cells could not be individually resolved and quantified due to their high packing density, whereas Hu-labeled neurons could be individually resolved and quantified.

Glial cell differentiation was determined by counting individual cells labeled for GFAP and APC within a fixed box size of 1,024 $\times$ 1,024 pixels at $\times 400$ magnification within the graft, divided by the total number of cells per sample box labeled with the human nuclear antigen hNu. Two randomly selected fields from the graft epicenter were counted in every 12th section in each animal for each label. The number of GFAP- and APC-labeled cells was counted in each grafted animal and divided by the mean number of hNu-labeled nuclei in the sampled field. Similar methods were used to quantify Ki67-labeled cells divided by the number of $\mathrm{hNu}^{+}$cells to obtain the percentage of Ki67 cells in the graft and at the C8 spinal cord level.

The ratio of migrating human cells expressing the glial marker hGFAP was determined by counting hGFAP and hNu double-labeled cells and dividing by the total number of $\mathrm{hNu}$ cells from $\times 400 \mathrm{Z}$-stack images taken from $\mathrm{C} 8$ coronal sections. The images were taken from 3 areas of the right-sided spinal cord white matter in which migrating, GFP-labeled cells were located: the dorsal columns, ventral white matter, and lateral white matter. The ratio of GFAP labeled glia in each area was averaged for each subject.

The number of GFP-labeled human axon profiles in gray matter of C8 coronal sections was counted from a $\times 400 \mathrm{Z}$-stack image in the medial central gray region including laminae $5-7$, which human axons predominately innervated; there was no human glial cell migration in this region. Quantification of 2 coronal sections was done, and an average axonal profile number was obtained by adding total axon numbers in each of 2 sections and dividing by 2 (the number of sampled sections per animal). In addition, the number of axons in the whole right side of spinal cord white matter from two T12 coronal sections per subject was manually counted and averaged. One subject that survived 18 months was excluded because of the presence of glial processes at the T12 level.

The density of 5-HT-labeled serotonergic axons penetrating grafts in lesion sites was quantified as previously described (53). Briefly, a series of 1-in-12 sections was labeled for 5-HT to visualize serotonergic axons. The rostral lesion/graft border was identifiable as a region of GFP-labeled grafted cells that also expressed NeuN (graft-derived neurons did not migrate from the lesion site). The number of pixels occupied by graft at $\times 100$ magnification was measured. The immunolabeled 5-HT axons within the graft were converted to black and white images, and the thresholding values of images were chosen, 
such that only immunolabeled axons were measured and light, nonspecific background labeling was not detected. The total number of 5-HT-labeled pixels was divided by the graft area to obtain the mean axon density per pixel of graft.

To assess synapse formation between human axonal terminals and host neurons, a segment of spinal cord at the T3 level from two 3-month surviving and two 18-month surviving animals was refixed with $4 \%$ paraformaldehyde and $0.25 \%$ glutaraldehyde overnight. Horizontal sections were cut on a vibratome set at 50- $\mu$ m intervals and processed for light-level observation of GFP immunolabeling using DAB and nickel chloride following the methods of Knott et al. (54). The host spinal cord gray matter containing GFP-labeled axons was then microscopically dissected and post-fixed with $1 \%$ osmium tetroxide, dehydrated, and embedded in Durcupan resin (SigmaAldrich). These samples were sectioned at a thickness of $60 \mathrm{~nm}$ using an ultramicrotome. Individual BDA-labeled axons or axonal terminals were located and assessed using an FEI 200KV Sphera microscope at the UCSD CryoElectron Microscopy Core Facility.

Statistics. For all quantification procedures, observers were blinded to the nature of the experimental manipulation. Comparisons among groups were tested by ANOVA (JMP software) at a designated significance level of $P<0.05$ and followed by Fisher's exact posthoc test between individual groups. Data are presented as the mean \pm SEM. For behavioral analysis, repeated-measures ANOVA was used to assess group differences over time, with Fisher's exact post-hoc test.
Study approval. All the animal experiments were approved by the IACUC of the VA-San Diego Healthcare System, and NIH guidelines for laboratory animal care and safety were strictly followed.

\section{Author contributions}

PL, SC, and MT designed the experiments. YW, LG, and EB performed the behavioral tests. DW and PL prepared cell cultures. YW, DW, and HK performed histology. PL, SC, YW, DW, and HK acquired data. PL, SC, and MT analyzed data and wrote the manuscript.

\section{Acknowledgments}

This work was supported by the Veterans Administration Gordon Mansfield Collaborative Consortium for Spinal Cord Injury Research (1I50RX001706-01); Veterans Adminstration Merit Review grants (1 I01 BX001252-01A2 and 1 I21 RX00084-01A1); the NIH (NS09881 and EB014986); the Craig H. Neilsen Foundation; the California Institute for Regenerative Medicine; the Dr. Miriam and Sheldon G. Adelson Medical Research Foundation; and the Bernard and Anne Spitzer Charitable Trust.

Address correspondence to: Mark H. Tuszynski, Department of Neurosciences, 0626, University of California, San Diego, La Jolla, California 92093, USA. Phone: 858.534.8857; Email: mtuszynski@ ucsd.edu.
1. Wictorin K, Brundin P, Gustavii B, Lindvall O, Björklund A. Reformation of long axon pathways in adult rat central nervous system by human forebrain neuroblasts. Nature. 1990;347(6293):556-558.

2. Lepore AC, Fischer I. Lineage-restricted neural precursors survive, migrate, and differentiate following transplantation into the injured adult spinal cord. Exp Neurol. 2005;194(1):230-242.

3. Tsuji $\mathrm{O}$, et al. Therapeutic potential of appropriately evaluated safe-induced pluripotent stem cells for spinal cord injury. Proc Natl Acad Sci US A. 2010;107(28):12704-12709.

4. Lu P, et al. Long-distance growth and connectivity of neural stem cells after severe spinal cord injury. Cell. 2012;150(6):1264-1273.

5. Lu P, et al. Long-distance axonal growth from human induced pluripotent stem cells after spinal cord injury. Neuron. 2014;83(4):789-796.

6 . Han X, et al. Forebrain engraftment by human glial progenitor cells enhances synaptic plasticity and learning in adult mice. Cell Stem Cell. 2013;12(3):342-353.

7. Blaya MO, Tsoulfas P, Bramlett HM, Dietrich WD. Neural progenitor cell transplantation promotes neuroprotection, enhances hippocampal neurogenesis, and improves cognitive outcomes after traumatic brain injury. Exp Neurol. 2015;264:67-81.

8. Kadoya K, et al. Spinal cord reconstitution with homologous neural grafts enables robust corticospinal regeneration. Nat Med.2016;22(5):479-487.

9. Nori S, et al. Grafted human-induced pluripotent stem-cell-derived neurospheres promote motor functional recovery after spinal cord injury in mice. Proc Natl Acad Sci U S A.
2011;108(40):16825-16830.

10. Iwai $\mathrm{H}$, et al. Allogeneic neural stem/progenitor cells derived from embryonic stem cells promote functional recovery after transplantation into injured spinal cord of nonhuman primates. Stem Cells Transl Med. 2015;4(7):708-719.

11. Semple BD, Blomgren K, Gimlin K, Ferriero DM, Noble-Haeusslein LJ. Brain development in rodents and humans: Identifying benchmarks of maturation and vulnerability to injury across species. Prog Neurobiol. 2013;106-107:1-16.

12. Nutt SE, et al. Caudalized human iPSC-derived neural progenitor cells produce neurons and glia but fail to restore function in an early chronic spinal cord injury model. Exp Neurol. 2013;248:491-503.

13. Brüstle $\mathrm{O}$, et al. Chimeric brains generated by intraventricular transplantation of fetal human brain cells into embryonic rats. Nat Biotechnol. 1998;16(11):1040-1044.

14. Windrem MS, et al. Fetal and adult human oligodendrocyte progenitor cell isolates myelinate the congenitally dysmyelinated brain. Nat Med. 2004;10(1):93-97.

15. Windrem MS, et al. Neonatal chimerization with human glial progenitor cells can both remyelinate and rescue the otherwise lethally hypomyelinated shiverer mouse. Cell Stem Cell. 2008;2(6):553-565.

16. Windrem MS, et al. A competitive advantage by neonatally engrafted human glial progenitors yields mice whose brains are chimeric for human glia. J Neurosci. 2014;34(48):16153-16161.

17. Wernig M, et al. Functional integration of embryonic stem cell-derived neurons in vivo. J Neurosci. 2004;24(22):5258-5268.
18. Nicholas CR, et al. Functional maturation of hPSC-derived forebrain interneurons requires an extended timeline and mimics human neural development. Cell Stem Cell. 2013;12(5):573-586.

19. Thomson JA, et al. Embryonic stem cell lines derived from human blastocysts. Science. 1998;282(5391):1145-1147.

20. Zhang SC, Wernig M, Duncan ID, Brüstle O, Thomson JA. In vitro differentiation of transplantable neural precursors from human embryonic stem cells. Nat Biotechnol. 2001;19(12):1129-1133.

21. Chang DJ, et al. Contralaterally transplanted human embryonic stem cell-derived neural precursor cells (ENStem-A) migrate and improve brain functions in stroke-damaged rats. Exp Mol Med. 2013;45:e53.

22. Khaing ZZ, et al. Assessing forelimb function after unilateral cervical spinal cord injury: novel forelimb tasks predict lesion severity and recovery. J Neurotrauma. 2012;29(3):488-498.

23. Lin R, Iacovitti L. Classic and novel stem cell niches in brain homeostasis and repair. Brain Res. 2015;1628(Pt B):327-342.

24. Barami K, Iversen K, Furneaux H, Goldman SA. Hu protein as an early marker of neuronal phenotypic differentiation by subependymal zone cells of the adult songbird forebrain. J Neurobiol. 1995;28(1):82-101.

25. Ince-Dunn G, et al. Neuronal Elav-like ( $\mathrm{Hu})$ proteins regulate RNA splicing and abundance to control glutamate levels and neuronal excitability. Neuron. 2012;75(6):1067-1080.

26. Greenlee JE, et al. Neuronal uptake of anti-Hu antibody, but not anti-Ri antibody, leads to cell death in brain slice cultures. J Neuroinflammation. 2014;11:160. 
27. Sarnat HB, Nochlin D, Born DE. Neuronal nuclear antigen (NeuN): a marker of neuronal maturation in early human fetal nervous system. Brain Dev. 1998;20(2):88-94.

28. Tamagno E, et al. H2O2 and 4-hydroxynonenal mediate amyloid beta-induced neuronal apoptosis by activating JNKs and p38MAPK. Exp Neurol. 2003;180(2):144-155.

29. Yamaguchi K, Goto N. Development of the human magnocellular red nucleus: a morphological study. Brain Dev. 2006;28(7):431-435.

30. Lukás Z, Dráber P, Bucek J, Dráberová E, Viklický V, Stasková Z. Expression of vimentin and glial fibrillary acidic protein in human developing spinal cord. Histochem J. 1989;21(12):693-701.

31. Zhou Q, Wang S, Anderson DJ. Identification of a novel family of oligodendrocyte lineage-specific basic helix-loop-helix transcription factors. Neuron. $2000 ; 25(2): 331-343$.

32. Lang J, et al. Adenomatous polyposis coli regulates oligodendroglial development. J Neurosci. 2013;33(7):3113-3130.

33. Bin JM, Harris SN, Kennedy TE. The oligodendrocyte-specific antibody 'CC1' binds Quaking 7. J Neurochem. 2016;139(2):181-186.

34. Reubinoff BE, et al. Neural progenitors from human embryonic stem cells. Nat Biotechnol. 2001;19(12):1134-1140.

35. Keith D, El-Husseini A. Excitation control: Balancing PSD-95 function at the synapse. Front Mol Neurosci. 2008;1:4.

36. Tyagarajan SK, Fritschy JM. Gephyrin: a master regulator of neuronal function? Nat Rev Neurosci. 2014;15(3):141-156.

37. Ribotta MG, Provencher J, Feraboli-Lohnherr D, Rossignol S, Privat A, Orsal D. Activation of locomotion in adult chronic spinal rats is achieved by transplantation of embryonic raphe cells reinnervating a precise lumbar level. J Neurosci. 2000;20(13):5144-5152.

38. Musienko P, et al. Controlling specific locomotor behaviors through multidimensional monoaminergic modulation of spinal circuitries. J Neurosci. 2011;31(25):9264-9278.

39. Lynskey JV, et al. Delayed intervention with transplants and neurotrophic factors supports recovery of forelimb function after cervical spinal cord injury in adult rats. J Neurotrauma. 2006;23(5):617-634.

40. Houle JD, Tessler A. Repair of chronic spinal cord injury. Exp Neurol. 2003;182(2):247-260.

41. Geisler FH, Dorsey FC, Coleman WP. Recovery of motor function after spinal-cord injury--a randomized, placebo-controlled trial with GM-1 ganglioside. N Engl J Med. 1991;324(26):1829-1838.

42. Fawcett JW, et al. Guidelines for the conduct of clinical trials for spinal cord injury as developed by the ICCP panel: spontaneous recovery after spinal cord injury and statistical power needed for therapeutic clinical trials. Spinal Cord. 2007;45(3):190-205.

43. Oh SK, Choi KH, Yoo JY, Kim DY, Kim SJ, Jeon SR. A phase III clinical trial showing limited efficacy of autologous mesenchymal stem cell therapy for spinal cord injury. Neurosurgery. 2016;78(3):436-447; discussion 447.

44. Chen $\mathrm{H}$, et al. Human-derived neural progenitors functionally replace astrocytes in adult mice. JClin Invest. 2015;125(3):1033-1042.

45. Tuszynski MH, et al. Neural stem cell dissemination after grafting to CNS injury sites. Cell. 2014;156(3):388-389.

46. Steward O, Sharp KG, Yee KM, Hatch MN, Bonner JF. Characterization of ectopic colonies that form in widespread areas of the nervous system with neural stem cell transplants into the site of a severe spinal cord injury. J Neurosci. 2014;34(42):14013-14021.

47. Low LK, Cheng HJ. Axon pruning: an essential step underlying the developmental plasticity of neuronal connections. Philos Trans R Soc Lond B Biol Sci. 2006;361(1473):1531-1544.

48. Pozniak CD, Radinovic S, Yang A, McKeon F, Kaplan DR, Miller FD. An anti-apoptotic role for the p53 family member, p73, during developmental neuron death. Science. 2000;289(5477):304-306.

49. Taylor L, Jones L, Tuszynski MH, Blesch A. Neurotrophin-3 gradients established by lentiviral gene delivery promote short-distance axonal bridging beyond cellular grafts in the injured spinal cord. J Neurosci. 2006;26(38):9713-9721.

50. Lu P, et al. Motor axonal regeneration after partial and complete spinal cord transection. J Neurosci. 2012;32(24):8208-8218.

51. Gensel JC, Tovar CA, Hamers FP, Deibert RJ, Beattie MS, Bresnahan JC. Behavioral and histological characterization of unilateral cervical spinal cord contusion injury in rats. J Neurotrauma. 2006;23(1):36-54.

52. Schallert T, Fleming SM, Leasure JL, Tillerson JL, Bland ST. CNS plasticity and assessment of forelimb sensorimotor outcome in unilateral rat models of stroke, cortical ablation, parkinsonism and spinal cord injury. Neuropharmacology. 2000;39(5):777-787.

53. Lu P, Jones LL, Tuszynski MH. BDNF-expressing marrow stromal cells support extensive axonal growth at sites of spinal cord injury. Exp Neurol. 2005;191(2):344-360.

54. Knott GW, Holtmaat A, Trachtenberg JT, Svoboda K, Welker E. A protocol for preparing GFPlabeled neurons previously imaged in vivo and in slice preparations for light and electron microscopic analysis. Nat Protoc. 2009;4(8):1145-1156. 